\title{
An analytical approach for systems of fractional differential equations by means of the innovative homotopy perturbation method
}

\author{
RAHMAT DARZI AND BAHRAM Agheli
}

\begin{abstract}
We have applied the new approach of homotopy perturbation method (NAHPM) for partial differential system equations featuring time-fractional derivative. The Caputo-type of fractional derivative is considered in this paper. A combination of NAHPM and multiple fractional power series form has been used the first time to present analytical solution. In order to illustrate the simplicity and ability of the suggested approach, some specific and clear examples have been given. All numerical calculations in this manuscript have been carried out with Mathematica.
\end{abstract}

\section{INTRODUCTION}

In this research work, it has been proposed that the new HPM based on the multiple fractional power series can be engaged to solution of partial differential system equations featuring time-fractional derivative (FPDSEs).

This system equation has frequently appeared in different fields of science and engineering such as; physics, optics, plasma physics, superconductivity and quantum mechanics [1].

There are some more books related to fractional calculus for interested readers $[2,3]$. It should be noted that there are no accurate analytical solutions for most fractional differential equations. Consequently, for such equations we have to employ some direct and iterative methods. Researchers have used various methods to solve systems equations in recent years. Some familiar methods are: Adomian's decomposition method $[4,5,6]$, homotopy perturbation method $[7,8]$, homotopy analysis method $[9,10,11]$ and so on $[12,13,14,15,16,17,18]$.

This paper is organized: in Section 2, fundamental idea of the new method is presented. We explained convergence of this method in section 3. In

2010 Mathematics Subject Classification. Primary: 14F35, 35R11, 34A34.

Key words and phrases. System of fractional differential equations, new homotopy perturbation method, Caputo derivative.

Full paper. Received 2 April 2018, revised 24 April 2018, accepted 14 May 2018, available online 25 June 2018. 
Section 4, the application of innovative HPM to FPDSEs is illustrated, and some numerical examples are presented. And conclusions are drawn in Section 5 .

\section{Fundamental idea of THE NeW Method}

In this part of the paper, we present and define Riemann-Liouville fractional integral and Caputo's fractional derivative that are presented [2]. Then the new approach of homotopy perturbation method (NAHPM) is introduced and explained in detail.

Definition 2.1. A real function $f(x), x>0$, is considered to be in the space $C_{\nu},(\nu \in R)$, if there exists a real number $n(>\nu)$, so that $f(x)=x^{n} f_{1}(x)$, where $f_{1}(x) \in C[0, \infty)$, and it is said to be in the space $C_{\nu}^{k}$ if and only if $f^{(k)} \in C_{\nu}, k \in N$.

Definition 2.2. The Riemann-Liouville fractional integral operator of order of $\alpha>0$, of a function $f \in C_{\nu}, \nu \geq-1$, is given by

$$
\begin{aligned}
& I_{a}^{\alpha} f(x)=\frac{1}{\Gamma(\alpha)} \int_{a}^{x}(x-r)^{\alpha-1} f(r) \mathrm{d} r, \\
& I^{\alpha} f(x)=I_{0}^{\alpha} f(x), \quad I^{0} f(x)=f(x) .
\end{aligned}
$$

Definition 2.3. The Caputo's fractional derivative of $f$ is defined as

$$
D^{\alpha} f(x)=I^{k-\alpha} D^{k} f(x)=\frac{1}{\Gamma(k-\alpha)} \int_{0}^{x}(x-r)^{k-\alpha-1} f^{(k)}(r) \mathrm{d} r, \quad x>0 .
$$

where, $f \in C_{-1}^{k}, k-1<\alpha \leq k$ and $k \in \mathbb{N}$.

Remark 2.1. For $k-1<\alpha \leq k, k \in \mathbb{N}, f \in C_{\nu}^{k}, \nu \geq-1$ and $x>0$, the following properties satisfy

(i) $D_{a}^{\alpha} I_{a}^{\alpha} f(x)=f(x)$,

(ii) $I_{a}^{\alpha} D_{a}^{\alpha} f(x)=f(x)-\sum_{j=0}^{k-1} f^{(j)}\left(a^{+}\right) \frac{(x-a)^{j}}{j !}$.

To describe the fundamental ideas of the NAHPM method for partial differential system equations featuring time-fractional derivative:

$$
D_{\tau}^{\mu} u_{i}+N_{i}\left(\zeta_{1}, \zeta_{2}, \ldots, \zeta_{k-1}, \tau, u_{1}, u_{2}, \ldots, u_{k}\right)=h_{i}\left(\zeta_{1}, \zeta_{2}, \ldots, \zeta_{k-1}, \tau\right),
$$

in which $n-1<\mu \leq n$ and $i=1, \ldots, k$, with the following initial conditions for $i=1,2, \ldots, k$ and $j=1,2, \ldots, n-1$ :

$$
u_{i}^{(j)}\left(\zeta_{1}, \zeta_{2}, \ldots, \zeta_{k-1}, \tau_{0}\right)=g_{i j}\left(\zeta_{1}, \zeta_{2}, \ldots, \zeta_{k-1}\right) .
$$

where $N_{1}, \ldots, N_{k}$ are nonlinear operators, which usually depend on the functions $u_{i}$ and derivatives, $D^{\mu}$ denotes that Caputo fractional and $h_{1}, \ldots, h_{k}$, are inhomogeneous term.

For the solution of (1), by using NAHPM, we make the under homotopy for $i=1,2, \ldots, k$ : 


$$
\begin{aligned}
& *(1-q)\left(D_{\tau}^{\mu} U_{i}-u_{i 0}\right) \\
& \quad+q\left(D_{\tau}^{\mu} U_{i}+N\left(\zeta_{1}, \zeta_{2}, \ldots, \zeta_{k-1}, \tau, u_{1}, u_{2}, \ldots, u_{k}\right)-h_{i}\right)=0
\end{aligned}
$$

or

$$
D_{\tau}^{\mu} U_{i}=u_{i 0}-p\left(u_{i 0}+N\left(\zeta_{1}, \zeta_{2}, \ldots, \zeta_{k-1}, \tau, u_{1}, u_{2}, \ldots, u_{k}\right)-h_{i}\right) .
$$

Using the inverse operator, $L^{-1}=I_{\tau}^{\mu}($.$) to both sides of (3), then we gain$

$$
\begin{aligned}
U_{i}\left(\zeta_{1}, \zeta_{2}, \ldots, \zeta_{k-1}, \tau_{0}\right)= & U_{i}\left(\zeta_{1}, \zeta_{2}, \ldots, \zeta_{k-1}, \tau_{0}\right) \\
& +I_{\tau}^{\mu} u_{i 0}-p I_{\tau}^{\mu}\left(u_{i 0}\left(\zeta_{1}, \zeta_{2}, \ldots, \zeta_{k-1}, \tau_{0}\right)\right. \\
& \left.+N_{i}\left(\zeta_{1}, \zeta_{2}, \ldots, \zeta_{k-1}, \tau, u_{1}, u_{2}, \ldots, u_{k}\right)-h_{i}\right),
\end{aligned}
$$

where

$$
U_{i}\left(\zeta_{1}, \zeta_{2}, \ldots, \zeta_{k-1}, \tau_{0}\right)=\sum_{j=0}^{n-1} g_{i j} \frac{\tau^{j}}{j !}, i=1, \ldots, k .
$$

Now assume we introduce the solution of (4) in the next form

$$
U_{i}=U_{i 0}+q U_{i 1}+q^{2} U_{i 2}+\cdots
$$

where $U_{i j}, i=1, \ldots, k, j=0,1,2,3, \ldots$ are functions which should be calculated.

Definition 2.4. A series expansion of the next form

$$
\sum_{n=0}^{\infty} c_{n}\left(\tau-\tau_{0}\right)^{n \mu}=c_{0}+c_{1}\left(\tau-\tau_{0}\right)^{\mu}+c_{2}\left(\tau-\tau_{0}\right)^{2 \mu}+\cdots
$$

for $0 \leq n-1<\mu \leq n, t \leq \tau_{0}$, is called fractional power series around $\tau=\tau_{0}$.

Definition 2.5. A series expansion of the below form

$$
\sum_{n=0}^{\infty} h_{n}(\zeta)\left(\tau-\tau_{0}\right)^{n \mu}, \quad 0 \leq n-1<\mu \leq n, \quad t \leq \tau_{0}
$$

is called multiple fractional power series around $\tau=\tau_{0}$.

Assume that the initial approximation of the solution of relation (1) is in the following form:

$$
u_{i 0}\left(\zeta_{1}, \zeta_{2}, \ldots, \zeta_{k-1}, \tau\right)=\sum_{j=0}^{\infty} a_{i j}\left(\zeta_{1}, \zeta_{2}, \ldots, \zeta_{k-1}\right) p_{j}(\tau), \quad i=1, \ldots, k
$$

where $a_{i j}(\zeta), i=1, \ldots, k, j=0,1,2,3, \ldots$, are unfamiliar coefficients, and $p_{j}(\tau), j=0,1,2,3, \ldots$, are particular functions.

It is deserving to consider that if $h(\zeta, \tau)$, and $u_{0}(\zeta, \tau)$ are analytic around $\tau=0$, then their Taylor series can be written as

$$
u_{0}(\zeta, \tau)=\sum_{k=0}^{\infty} a_{k}(\zeta) \tau^{k \mu}
$$


With considering (4) and substituting (6) and (7) into that and equating the coefficients of the same power $q$, for $i=1, \ldots, k$, with

$$
\begin{aligned}
& q^{0}: U_{i 0}\left(\zeta_{1}, \zeta_{2}, \ldots, \zeta_{k-1}, \tau\right) \\
& =U_{i}\left(\zeta_{1}, \zeta_{2}, \ldots, \zeta_{k-1}, \tau_{0}\right)+\sum_{j=0}^{\infty} a_{i j}(\zeta) I_{\tau}^{\mu}\left(p_{j}(\tau)\right) \\
& q^{1}: U_{i 1}\left(\zeta_{1}, \zeta_{2}, \ldots, \zeta_{k-1}, \tau\right)=-\sum_{j=0}^{\infty} a_{i j}(\zeta) I_{\tau}^{\mu}\left(p_{j}(\tau)\right) \\
& \quad-I_{\tau}^{\mu}\left(N_{i}\left(\zeta_{1}, \zeta_{2}, \ldots, \zeta_{k-1}, \tau, U_{10}, U_{20}, \ldots, U_{k 0}\right)-h_{i}\right) \\
& q^{2}: U_{i 2}\left(\zeta_{1}, \zeta_{2}, \ldots, \zeta_{k-1}, \tau\right) \\
& =-I_{\tau}^{\mu}\left(N_{i}\left(\zeta_{1}, \zeta_{2}, \ldots, \zeta_{k-1, \tau}, U_{10}, U_{20}, \ldots, U_{k 0}, U_{11}, U_{21}, \ldots, U_{k 1}\right)\right), \\
& \left.\left.\quad \vdots \quad U_{10}, U_{20}, \ldots, U_{k 0}, U_{1 j-1}, \ldots, U_{2 j-1}, \ldots, U_{k j-1}\right)\right) \\
& q^{j}: U_{i j}\left(\zeta_{1}, \zeta_{2}, \ldots, \zeta_{k-1}, \tau\right)=I_{\tau}^{\mu}\left(N _ { i } \left(\zeta_{1}, \zeta_{2}, \ldots, \zeta_{k-1}, \tau,\right.\right.
\end{aligned}
$$

By solving these equations in such a manner that

$$
U_{i 1}\left(\zeta_{1}, \zeta_{2}, \ldots, \zeta_{k-1}, \tau\right)=0, i=1, \ldots, k,
$$

then relations (9) yield to

$$
U_{i 2}\left(\zeta_{1}, \zeta_{2}, \ldots, \zeta_{k-1}, \tau\right)=U_{i 3}\left(\zeta_{1}, \zeta_{2}, \ldots, \zeta_{k-1}, \tau\right)=\ldots=0 .
$$

Therefore, the numerical analytical solution may be gained as follows:

$$
\begin{aligned}
& U_{i}\left(\zeta_{1}, \zeta_{2}, \ldots, \zeta_{k-1}, \tau\right)=U_{i 0}\left(\zeta_{1}, \zeta_{2}, \ldots, \zeta_{k-1}, \tau\right) \\
& =U_{i}\left(\zeta_{1}, \zeta_{2}, \ldots, \zeta_{k-1}, \tau_{0}\right)+\sum_{j=0}^{\infty} a_{i j}(\zeta) I_{\tau}^{\mu}\left(p_{j}(\tau)\right), i=1, \ldots, k .
\end{aligned}
$$

It should be noted that if $h_{i}\left(\zeta_{1}, \zeta_{2}, \ldots, \zeta_{k-1}, \tau\right), u_{i}\left(\zeta_{1}, \zeta_{2}, \ldots, \zeta_{k-1}, \tau\right)$, are analytic around $\tau=\tau_{0}$, then Taylor series can be written as

$$
\begin{aligned}
u_{i 0}\left(\zeta_{1}, \zeta_{2}, \ldots, \zeta_{k-1}, \tau\right) & =\sum_{j=0}^{\infty} a_{i j}\left(\zeta_{1}, \zeta_{2}, \ldots, \zeta_{k-1}\right)\left(\tau-\tau_{0}\right)^{k \mu}, \\
h_{i}\left(\zeta_{1}, \zeta_{2}, \ldots, \zeta_{k-1}, \tau\right) & =\sum_{j=0}^{\infty} a_{i j}^{\star}\left(\zeta_{1}, \zeta_{2}, \ldots, \zeta_{k-1}\right)\left(\tau-\tau_{0}\right)^{k \mu},
\end{aligned}
$$

can be used in relations (9), where $a_{i j}\left(\zeta_{1}, \zeta_{2}, \ldots, \zeta_{k-1}\right)$ are unknown coefficients which must be computed, and $a_{i j}^{\star}\left(\zeta_{1}, \zeta_{2}, \ldots, \zeta_{k-1}\right), i=1, \ldots, k$, and $j=0,1,2, \ldots$, are known ones. 


\section{Convergence analysis}

A large number of problems can be treated by NAHPM through applying the methodology that has been elaborated in the previous sections.

Theorem 3.1. Presume that $S$ and $T$ are Banach spaces and $\mathcal{A}: S \rightarrow T$ is a contractive nonlinear mapping which is

$$
\forall v, v^{\star} \in S, \quad\left\|\mathcal{A}(v)-\mathcal{A}\left(v^{\star}\right)\right\| \leq \lambda\left\|v-v^{\star}\right\|, \quad 0<\lambda<1 .
$$

Then due to Banach's fixed point theorem $\mathcal{A}$ has a unique fixed point $u$, which is $\mathcal{A}(u)=u$.

Assume that the sequence provided by new HPM is stated that

$$
w_{k}=\mathcal{A}\left(w_{k-1}\right), \quad w_{k-1}=\sum_{i=0}^{k-1} v_{i}, \quad k=1,2, \ldots,
$$

assume that $w_{0}=v_{0} \in B_{r}(v)$, where $B_{r}(v)=\left\{v^{\star} \in S ; \quad\left\|v^{\star}-v\right\|<r\right\}$, then we have

(i) $w_{k} \in B_{r}(v)$,

(ii) $\lim _{k \rightarrow \infty} w_{k}=v$.

Proof. (i) By inductive way featuring $k=1$

$$
\left\|w_{1}-v\right\|=\left\|\mathcal{A}\left(w_{0}\right)-\mathcal{A}(v)\right\| \leq \lambda\left\|v_{0}-v\right\| .
$$

Allow that $\left\|w_{k-1}-v\right\| \leq \lambda^{k-1}\left\|v_{0}-v\right\|$, while induction hypothesis, hence $\left\|w_{k}-v\right\|=\left\|\mathcal{A}\left(w_{k-1}\right)-\mathcal{A}(v)\right\| \leq \lambda\left\|v_{k-1}-v\right\| \leq\left\|w_{k-1}-v\right\| \leq \lambda^{k}\left\|v_{0}-v\right\|$. So

$$
\left\|w_{k}-v\right\| \leq \lambda^{k}\left\|v_{0}-v\right\| \leq \lambda^{k} r<r,
$$

in this manner $w_{k} \in B_{r}(v)$.

(ii) Due to $\left\|w_{k}-v\right\| \leq \lambda^{k}\left\|v_{0}-v\right\|$ and $\lim _{k \rightarrow \infty} \lambda^{k}=0, \lim _{k \rightarrow \infty}\left\|w_{k}-v\right\|=0$, that is, $\lim _{k \rightarrow \infty} w_{k}=v$.

\section{Test eXAmples}

Now, we apply NAHPM based on the multiple fractional power series to solve FPDSEs. All of the plots and computations for this equations have been done with Mathematica ${ }^{\circledR}$.

Example 4.1. We purpose the following FPDSEs:

$$
\left\{\begin{array}{l}
D_{t}^{\mu} u-v u_{x x}-u v_{x x}=\frac{3 e^{x} t^{2 \mu}}{\Gamma(2 \mu)}+\frac{2 t^{\mu} \sin (x)}{\Gamma(\mu)}, \quad 1<\mu \leq 2, \\
D_{t}^{\mu} v-v v_{x x}+u u_{x x}=-\frac{9 e^{2 x} t^{4 \mu}}{\Gamma(2 \mu)^{2}}-\frac{t^{4 \mu} \sin ^{2}(x)}{\Gamma(2 \mu)^{2}}+\frac{t^{2 \mu} \sin (x)}{\Gamma(2 \mu)}+\frac{6 e^{x} t^{\mu}}{\Gamma(\mu)}
\end{array}\right.
$$


(II)

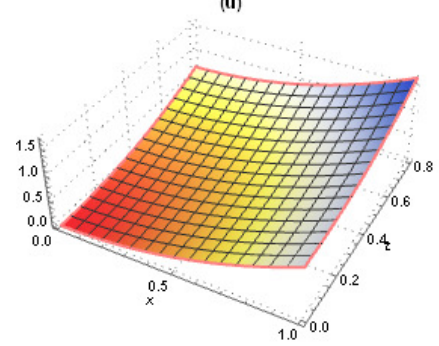

(v)

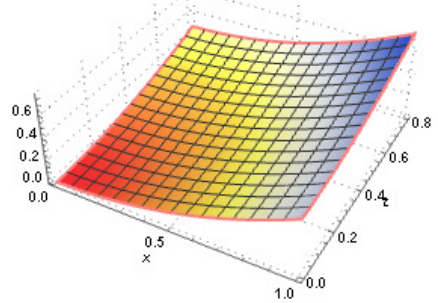

FiguRE 1. Approximate result of test example 4.1.

The estimate answers featuring $\mu=2$ acquired for several amounts of $x$ and $t$ applying NAHPM, is shown in Table 1 . featuring the primary conditions:

$$
\begin{aligned}
& u(x, 0)=\frac{x^{2}}{\Gamma(\mu+1)}, \quad u_{t}(x, 0)=0, \\
& v(x, 0)=\frac{x^{2}}{2 \Gamma(\mu+1)}, \quad v_{t}(x, 0)=0 .
\end{aligned}
$$

TABle 1. Approximate result of test example 4.1.

\begin{tabular}{|c|c|c|c|}
\hline$t$ & $x$ & $u_{N A H P M}=u_{\text {Exact }}$ & $v_{N A H P M}=v_{\text {Exact }}$ \\
\hline \multirow{3}{*}{0.1} & 0.50 & 0.130000 & 0.065000 \\
& 0.75 & 0.286250 & 0.143125 \\
& 1.00 & 0.505000 & 0.252500 \\
\hline \multirow{4}{*}{0.3} & 0.50 & 0.170000 & 0.085000 \\
& 0.75 & 0.326250 & 0.163125 \\
& 1.00 & 0.545000 & 0.272500 \\
\hline \multirow{3}{*}{0.5} & 0.50 & 0.250000 & 0.125000 \\
& 0.75 & 0.406250 & 0.203125 \\
& 1.00 & 0.625000 & 0.312500 \\
\hline
\end{tabular}

Assume

$$
\begin{aligned}
& u_{0}(x, t)=\sum_{k=0}^{m} a_{k}(x) t^{k \mu}, \quad U(x, 0)=g(x), \\
& v_{0}(x, t)=\sum_{k=0}^{m} b_{k}(x) t^{k \mu}, \quad V(x, 0)=h(x) .
\end{aligned}
$$


Solving equations (11)-(12) for $U_{1}(x, t), V_{1}(x, t)$ guidance to the following conclusion:

$$
\begin{aligned}
& U_{1}(x, t)=t^{\mu}\left(\frac{1}{\mu \Gamma(\mu)}-\frac{a_{0}(x)}{\mu \Gamma(\mu)}\right)+ \\
& t^{2 \mu}\left(-\frac{1}{\mu^{2} \Gamma(\mu) \Gamma(2 \mu)}+\frac{a_{0}(x)}{\mu \Gamma(\mu) \Gamma(2 \mu+1)}-\frac{\sqrt{\pi} 2^{-2 \mu} a_{1}(x)}{\Gamma\left(\mu+\frac{1}{2}\right)}+\right. \\
& \left.\frac{x^{2} a_{0}^{\prime \prime}(x)}{4 \mu^{2} \Gamma(\mu) \Gamma(2 \mu)}+\frac{x^{2} b_{0}^{\prime \prime}(x)}{\mu \Gamma(\mu) \Gamma(2 \mu+1)}+\frac{b_{0}(x)}{\mu^{2} \Gamma(\mu) \Gamma(2 \mu)}\right)+\cdots \text {. } \\
& V_{1}(x, t)=t^{\mu}\left(\frac{1}{2 \mu \Gamma(\mu)}-\frac{b_{0}(x)}{\mu \Gamma(\mu)}\right)+ \\
& t^{2 \mu}\left(\frac{3}{4 \mu^{2} \Gamma(\mu) \Gamma(2 \mu)}-\frac{a_{0}(x)}{\mu^{2} \Gamma(\mu) \Gamma(2 \mu)}+\frac{b_{0}(x)}{\mu \Gamma(\mu) \Gamma(2 \mu+1)}-\right. \\
& \left.-\frac{x^{2} a_{0}^{\prime \prime}(x)}{\mu \Gamma(\mu) \Gamma(2 \mu+1)}+\frac{x^{2} b_{0}^{\prime \prime}(x)}{4 \mu^{2} \Gamma(\mu) \Gamma(2 \mu)}+\frac{\sqrt{\pi} 2^{-2 \mu} b_{1}(x)}{\Gamma\left(\mu+\frac{1}{2}\right)}\right)+\cdots .
\end{aligned}
$$

When $U_{1}(x, t)$ and $V_{1}(x, t)$ are vanished, the coefficients $a_{k}(x), k=1,2,3, \ldots$, will be gained is state as:

$$
a_{0}(x)=1, \quad a_{1}(x)=0, \quad a_{2}(x)=0, \ldots
$$

and

$$
b_{0}(x)=\frac{1}{2}, \quad b_{1}(x)=0, \quad b_{2}(x)=0, \ldots
$$

This outcomes that

$$
\begin{gathered}
u(x, t)=U_{0}(x, t)=\frac{t^{\mu}+x^{2}}{\Gamma(\mu+1)}, \\
v(x, t)=V_{0}(x, t)=\frac{t^{\mu}+x^{2}}{2 \Gamma(\mu+1)} .
\end{gathered}
$$

In Figure 4.1, we may view the estimate answers featuring $\mu=2$ which is concluded for several amounts of $t$ and $x$ utilizing NAHPM.

Example 4.2. Next, we consider the system of coupled fractional equations [19]:

$$
\left\{\begin{array}{l}
D_{t}^{\mu} u-u_{x x}+u u_{x}+(u v)_{x}=\frac{\left(2 x^{3}+1\right) t^{2 \mu}}{\Gamma(\mu+1)}-\frac{2 t^{\mu}}{\Gamma(\mu+1)}+x^{2}, 0<\mu \leq 1 \\
D_{t}^{\mu} v-v_{x x}+v v_{x}-(u v)_{x}=\frac{\left(\frac{1}{x^{3}}+1\right) t^{2 \mu}}{\Gamma(\mu+1)}-\frac{2 t^{\mu}}{x^{3} \Gamma(\mu+1)}+\frac{1}{x}
\end{array}\right.
$$

along with:

$$
u(x, 0)=0, \quad v(x, 0)=0 .
$$


With considering $U_{1}(x, t)$ and $V_{1}(x, t)$, the result shows

$$
\begin{gathered}
U_{1}(x, t)=t^{\mu}\left(\frac{x^{2}}{\mu \Gamma(\mu)}-\frac{a_{0}(x)}{\mu \Gamma(\mu)}\right)+ \\
t^{2 \mu}\left(\frac{\sqrt{\pi} 2^{-2 \mu} a_{0}^{\prime \prime}(x)}{\mu \Gamma(\mu) \Gamma\left(\mu+\frac{1}{2}\right)}-\frac{\sqrt{\pi} 2^{-2 \mu} a_{1}(x)}{\Gamma\left(\mu+\frac{1}{2}\right)}-\frac{\sqrt{\pi} 2^{1-2 \mu}}{\mu \Gamma(\mu) \Gamma\left(\mu+\frac{1}{2}\right)}\right)+ \\
t^{3 \mu}\left(\frac{2 \Gamma(2 \mu)}{\Gamma(\mu) \Gamma(3 \mu+1)}+\frac{4 x^{3} \Gamma(2 \mu)}{\Gamma(\mu) \Gamma(3 \mu+1)}-\frac{a_{2}(x) \Gamma(2 \mu+1)}{\Gamma(3 \mu+1)}-\right. \\
\frac{a_{0}(x) \Gamma(2 \mu+1) a_{0}^{\prime}(x)}{\mu^{2} \Gamma(\mu)^{2} \Gamma(3 \mu+1)}-\frac{\Gamma(2 \mu+1) b_{0}(x) a_{0}^{\prime}(x)}{\mu^{2} \Gamma(\mu)^{2} \Gamma(3 \mu+1)}- \\
\left.\frac{a_{0}(x) \Gamma(2 \mu+1) b_{0}^{\prime}(x)}{\mu^{2} \Gamma(\mu)^{2} \Gamma(3 \mu+1)}+\frac{\Gamma(\mu+1) a_{1}^{\prime \prime}(x)}{\Gamma(3 \mu+1)}\right)+\cdots
\end{gathered}
$$

$(20)$

$$
\begin{gathered}
V_{1}(x, t)=t^{\mu}\left(\frac{1}{\mu x \Gamma(\mu)}-\frac{b_{0}(x)}{\mu \Gamma(\mu)}\right)+ \\
t^{2 \mu}\left(\frac{\sqrt{\pi} 2^{-2 \mu} b_{0}^{\prime \prime}(x)}{\mu \Gamma(\mu) \Gamma\left(\mu+\frac{1}{2}\right)}-\frac{\sqrt{\pi} 2^{-2 \mu} b_{1}(x)}{\Gamma\left(\mu+\frac{1}{2}\right)}-\frac{\sqrt{\pi} 2^{1-2 \mu}}{\mu x^{3} \Gamma(\mu) \Gamma\left(\mu+\frac{1}{2}\right)}\right)+ \\
t^{3 \mu}\left(\frac{2 \Gamma(2 \mu)}{\Gamma(\mu) \Gamma(3 \mu+1)}+\frac{2 \Gamma(2 \mu)}{x^{3} \Gamma(\mu) \Gamma(3 \mu+1)}-\frac{\Gamma(2 \mu+1) b_{2}(x)}{\Gamma(3 \mu+1)}-\right. \\
\frac{\Gamma(2 \mu+1) b_{0}(x) a_{0}^{\prime}(x)}{\mu^{2} \Gamma(\mu)^{2} \Gamma(3 \mu+1)}-\frac{a_{0}(x) \Gamma(2 \mu+1) b_{0}^{\prime}(x)}{\mu^{2} \Gamma(\mu)^{2} \Gamma(3 \mu+1)}- \\
\left.\frac{\Gamma(2 \mu+1) b_{0}(x) b_{0}^{\prime}(x)}{\mu^{2} \Gamma(\mu)^{2} \Gamma(3 \mu+1)}+\frac{\Gamma(\mu+1) b_{1}^{\prime \prime}(x)}{\Gamma(3 \mu+1)}\right)+\cdots
\end{gathered}
$$
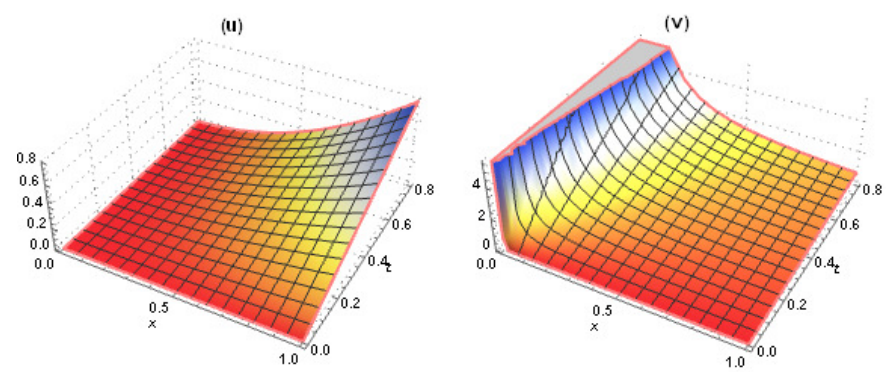

Figure 2. The estimate solution for $\mu=1$. 
Considering the hypothesis $U_{1}(x, t)=0, V_{1}(x, t)=0$, coefficients $a_{k}(x)$, $b_{k}(x), k=1,2,3, \ldots$, will be determined as follows:

$$
a_{0}(x)=x^{2}, \quad a_{1}(x)=0, \quad a_{2}(x)=0, \quad \ldots,
$$

and

$$
b_{0}(x)=\frac{1}{x}, \quad b_{1}(x)=0, \quad b_{2}(x)=0, \quad \ldots
$$

Therefore we provide the solution of (17) which that comes next:

$$
u(x, t)=\frac{x^{2} t^{\mu}}{\mu \Gamma(\mu)}, \quad v(x, t)=\frac{t^{\mu}}{x \Gamma(\mu+1)} .
$$

In Figure 2, we can view the approximate answers featuring $\mu=1$.

In Table 2 , we may view the approximate answers featuring $\mu=1$, which is concluded for several amounts of $t$ and $x$ applying NAHPM.

TABLE 2. Approximate result of test example 4.2.

\begin{tabular}{|c|c|c|c|}
\hline$t$ & $x$ & $u_{N A H P M}=u_{\text {Exact }}$ & $v_{N A H P M}=v_{\text {Exact }}$ \\
\hline \multirow{3}{*}{0.1} & 0.50 & 0.02500 & 0.200000 \\
& 0.75 & 0.05625 & 0.133333 \\
& 1.00 & 0.10000 & 0.100000 \\
\hline \multirow{3}{*}{0.3} & 0.50 & 0.07500 & 0.600000 \\
& 0.75 & 0.16875 & 0.400000 \\
& 1.00 & 0.30000 & 0.300000 \\
\hline \multirow{3}{*}{0.5} & 0.50 & 0.12500 & 1.000000 \\
& 0.75 & 0.28125 & 0.666667 \\
& 1.00 & 0.50000 & 0.500000 \\
\hline
\end{tabular}

Example 4.3. We choose the non-linear system of inhomogeneous FPDEs:

$$
\left\{\begin{array}{l}
D_{t}^{\mu} u-w_{x} v_{x}-\frac{1}{2} w_{x} u_{x x}=-\frac{2 \mu t^{\mu}}{\Gamma(\mu+1)}-\frac{4 x^{2}}{\Gamma(2 \mu)^{2}}-\frac{2 x}{\Gamma(2 \mu)^{2}}, \quad 0<\mu \leq 1, \\
D_{t}^{\mu} v-w_{x} u_{x x}=\frac{2 \mu t^{\mu}}{\Gamma(\mu+1)}-\frac{4 x}{\Gamma(2 \mu)^{2}}, \\
D_{t}^{\mu} w-u_{x x}-v_{x} w_{x}=-\frac{2}{\Gamma(2 \mu)}-\frac{2 \mu t^{\mu}}{\Gamma(\mu+1)}-\frac{4 x^{2}}{\Gamma(2 \mu)^{2}},
\end{array}\right.
$$

with the primary conditions:

$$
u(x, 0)=\frac{1+x^{2}}{\Gamma(2 \mu)}, \quad v(x, 0)=\frac{x^{2}+2}{\Gamma(2 \mu)}, \quad w(x, 0)=\frac{3 x^{2}}{\Gamma(2 \mu)} .
$$

Assume

$$
u_{0}(x, t)=\sum_{k=0}^{m} a_{k}(x) t^{k \mu}, \quad v_{0}(x, t)=\sum_{k=0}^{m} b_{k}(x) t^{k \mu}, \quad w_{0}(x, t)=\sum_{k=0}^{m} c_{k}(x) t^{k \mu},
$$


and $U(x, 0)=g(x), V(x, 0)=h(x), W(x, 0)=k(x)$. With considering $U_{1}(x, t), V_{1}(x, t)$ and $W_{1}(x, t)$, we have

$$
\begin{aligned}
& U_{1}(x, t)=\frac{a_{0}(x) t^{\mu}}{\mu \Gamma(\mu)}+t^{2 \mu}\left(-\frac{\sqrt{\pi} 2^{1-2 \mu}}{\Gamma(\mu) \Gamma\left(\mu+\frac{1}{2}\right)}-\frac{\sqrt{\pi} 2^{-2 \mu} a_{1}(x)}{\Gamma\left(\mu+\frac{1}{2}\right)}+\right. \\
& \frac{\sqrt{\pi} 2^{1-2 \mu} x b_{0}^{\prime}(x)}{\mu \Gamma(\mu) \Gamma(2 \mu) \Gamma\left(\mu+\frac{1}{2}\right)}+\frac{\sqrt{\pi} 2^{-2 \mu} c_{0}^{\prime}(x)}{\mu \Gamma(\mu) \Gamma(2 \mu) \Gamma\left(\mu+\frac{1}{2}\right)}+ \\
& \left.\frac{\sqrt{\pi} 2^{-2 \mu} x a_{0}^{\prime \prime}(x)}{\mu \Gamma(\mu) \Gamma(2 \mu) \Gamma\left(\mu+\frac{1}{2}\right)}+\frac{\sqrt{\pi} 2^{1-2 \mu} x c_{0}^{\prime}(x)}{\mu \Gamma(\mu) \Gamma(2 \mu) \Gamma\left(\mu+\frac{1}{2}\right)}\right)+\cdots, \\
& V_{1}(x, t)=-\frac{b_{0}(x) t^{\mu}}{\mu \Gamma(\mu)}+t^{2 \mu}\left(\frac{\sqrt{\pi} 2^{1-2 \mu}}{\Gamma(\mu) \Gamma\left(\mu+\frac{1}{2}\right)}-\frac{\sqrt{\pi} 2^{-2 \mu} b_{1}(x)}{\Gamma\left(\mu+\frac{1}{2}\right)}+\right. \\
& \left.\frac{\sqrt{\pi} 2^{1-2 \mu} x a_{0}^{\prime \prime}(x)}{\mu \Gamma(\mu) \Gamma(2 \mu) \Gamma\left(\mu+\frac{1}{2}\right)}+\frac{\sqrt{\pi} 2^{1-2 \mu} c_{0}^{\prime}(x)}{\mu \Gamma(\mu) \Gamma(2 \mu) \Gamma\left(\mu+\frac{1}{2}\right)}\right)+\cdots, \\
& W_{1}(x, t)=-\frac{c_{0}(x) t^{\mu}}{\mu \Gamma(\mu)}+\frac{\Gamma(2 \mu+1)^{2} \Gamma(6 \mu+1) t^{7 \mu} b_{2}^{\prime}(x) c_{2}^{\prime}(x)}{\Gamma(3 \mu+1)^{2} \Gamma(7 \mu+1)}+ \\
& t^{2 \mu}\left(-\frac{\sqrt{\pi} 2^{1-2 \mu}}{\Gamma(\mu) \Gamma\left(\mu+\frac{1}{2}\right)}-\frac{\sqrt{\pi} 2^{-2 \mu} c_{1}(x)}{\Gamma\left(\mu+\frac{1}{2}\right)}+\frac{\sqrt{\pi} 2^{1-2 \mu} x b_{0}^{\prime}(x)}{\mu \Gamma(\mu) \Gamma(2 \mu) \Gamma\left(\mu+\frac{1}{2}\right)}+\right. \\
& \left.\frac{\sqrt{\pi} 2^{-2 \mu} a_{0}^{\prime \prime}(x)}{\mu \Gamma(\mu) \Gamma\left(\mu+\frac{1}{2}\right)}+\frac{\sqrt{\pi} 2^{1-2 \mu} x c_{0}^{\prime}(x)}{\mu \Gamma(\mu) \Gamma(2 \mu) \Gamma\left(\mu+\frac{1}{2}\right)}\right)+\cdots .
\end{aligned}
$$

Accordingly, by vanishing of $U_{1}(x, t), V_{1}(x, t)$ and $W_{1}(x, t)$ the coefficients $a_{k}(x), b_{k}(x)$ and $c_{k}(x), k=1,2, \ldots$, will be gained:

$$
a_{0}(x)=0, \quad a_{1}(x)=-\frac{2}{\Gamma(\mu)}, \quad a_{2}(x)=0, \quad \ldots,
$$

and

$$
b_{0}(x)=0, \quad b_{1}(x)=\frac{2}{\Gamma(\mu)}, \quad b_{2}(x)=0,
$$

and

$$
c_{0}(x)=0, \quad c_{1}(x)=-\frac{2}{\Gamma(\mu)}, \quad c_{2}(x)=0, \quad \ldots
$$

Therefore we obtain approximate solution of Eq.(22)

$$
\begin{aligned}
& u(x, t)=U_{0}(x, t)=\frac{1}{\Gamma(2 \mu)}-\frac{\sqrt{\pi} 2^{1-2 \mu} t^{2 \mu}}{\Gamma(\mu) \Gamma\left(\mu+\frac{1}{2}\right)}+\frac{x^{2}}{\Gamma(2 \mu)}, \\
& v(x, t)=V_{0}(x, t)=\frac{2}{\Gamma(2 \mu)}+\frac{\sqrt{\pi} 2^{1-2 \mu} t^{2 \mu}}{\Gamma(\mu) \Gamma\left(\mu+\frac{1}{2}\right)}+\frac{x^{2}}{\Gamma(2 \mu)},
\end{aligned}
$$



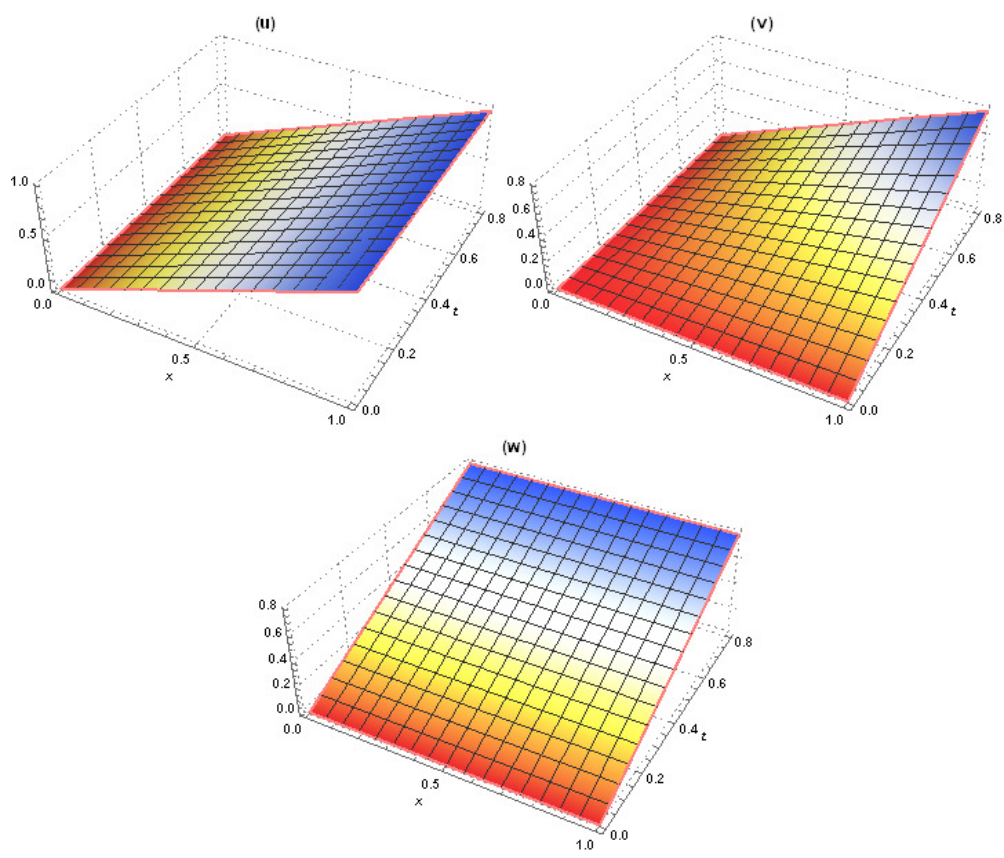

Figure 3. The estimate solution for $\mu=1$.

and

$$
w(x, t)=W_{0}(x, t)=\frac{3}{\Gamma(2 \mu)}-\frac{\sqrt{\pi} 2^{1-2 \mu} t^{2 \mu}}{\Gamma(\mu) \Gamma\left(\mu+\frac{1}{2}\right)}+\frac{x^{2}}{\Gamma(2 \mu)} .
$$

We can see the analytical answers toward $\mu=1$, in figure 3 .

The analytical answers featuring $\mu=1$ acquired for several amounts of $x$, and $t$ applying NAHPM, can be seen in Table 3.

TABle 3. Approximate result of test example 4.3.

\begin{tabular}{|c|c|c|c|c|}
\hline$t$ & $x$ & $u_{N A H P M}=u_{\text {Exact }}$ & $v_{N A H P M}=v_{\text {Exact }}$ & $w_{\text {NAHPM }}=w_{\text {Exact }}$ \\
\hline \multirow{3}{*}{0.1} & 0.50 & 1.2400 & 2.2600 & 3.2400 \\
& 0.75 & 1.5525 & 2.5725 & 3.5525 \\
& 1.00 & 1.9900 & 3.0100 & 3.9900 \\
\hline \multirow{3}{*}{0.3} & 0.50 & 1.1600 & 2.3400 & 3.1600 \\
& 0.75 & 1.4725 & 2.6525 & 3.4725 \\
& 1.00 & 1.9100 & 3.0900 & 3.9100 \\
\hline \multirow{4}{*}{0.5} & 0.50 & 1.0000 & 2.5000 & 3.0000 \\
& 0.75 & 1.4725 & 2.6525 & 3.4725 \\
& 1.00 & 1.7500 & 3.2500 & 3.7500 \\
\hline
\end{tabular}




\section{Conclusion}

In this paper, we have successfully applied NAHPM to obtain series solution of partial differential system equations featuring time-fractional derivative. The result indicated that a few iteration of NAHPM will result in some solution.

Finally, it should be added that the suggested approach has the potentials to be applied in solving other similar nonlinear problems in partial differential equations of fractional order.

\section{REFERENCES}

[1] D. Baleanu, A. C. Luo, Discontinuity and Complexity in Nonlinear Physical Systems, J. T. Machado (Ed.), Springer, 2014.

[2] A. A. Kilbas, H. M. Srivastava, J. J. Trujillo, Theory and application of fractional differential equations, Elsevier B. V., Netherlands, 2006.

[3] K. S. Miller, B. Ross, An introduction to the fractional calculus and fractional differential equation, John Wiley and Sons, New York, 1993.

[4] S. Momani, Z. Odibat, Numerical approach to differential equations of fractional order, Journal of Computational and Applied Mathematics, 207 (1) (2007), 96-110.

[5] J. Biazar, H. Ghazvini, He's variational iteration method for solving linear and nonlinear systems of ordinary differential equations, Applied Mathematics and Computation, 191 (1) (2007), 287-297.

[6] J. Biazar, K. Hosseini, An Effective Modification of Adomian Decomposition Method for Solving EmdenâA ŞFowler Type Systems, National Academy Science Letters, 40 (4) (2017), 285-290.

[7] D. D. Ganji, A. Sadighi, Application of He's homotopy-perturbation method to nonlinear coupled systems of reaction-diffusion equations, International Journal of Nonlinear Sciences and Numerical Simulation, 7 (4) (2006), 411-418.

[8] J. Biazar, M. Eslami, H. Ghazvini, Homotopy perturbation method for systems of partial differential equations, International Journal of Nonlinear Sciences and Numerical Simulation, 8 (3) (2007), 413-418.

[9] M. Zurigat, S. Momani, Z. Odibat, A. Alawneh, The homotopy analysis method for handling systems of fractional differential equations, Applied Mathematical Modelling, 34 (1) (2010), 24-35.

[10] M. Zurigat, S. Momani, A. Alawneh, Analytical approximate solutions of systems of fractional algebraicâĂŞdifferential equations by homotopy analysis method, Computers \& Mathematics with Applications, 59 (3) (2010), 1227-1235.

[11] J. Biazar, R. Ansari, K. Hosseini, M. Aligoli, Homotopy perturbation method for solving linear and non-linear systems of PDEs. Journal of Nature Science and Sustainable Technology, 4 (3) (2011), 185-196.

[12] I. A. H. Hassan, Application to differential transformation method for solving systems of differential equations, Applied Mathematical Modelling, 32 (12) (2008), 2552-2559. 
[13] M. Tatari, M. Dehghan, Improvement of He's variational iteration method for solving systems of differential equations, Computers \& Mathematics with Applications, 58 (11) (2009), 2160-2166.

[14] K. Hosseini, R. Ansari, P. Gholamin, Exact solutions of some nonlinear systems of partial differential equations by using the first integral method, Journal of Mathematical Analysis and Applications, 387 (2) (2012), 807-814.

[15] K. Hosseini, J. Biazar, R. Ansari, P. Gholamin, A new algorithm for solving differential equations, Mathematical Methods in the Applied Sciences, 35 (9) (2012), 993-999.

[16] J. Biazar, K. Hosseini, Analytic approximation of Volterra's population model. Journal of Applied Mathematics, Statistics and Informatics, 13 (1) (2017), 5-17.

[17] J. Biazar, K. Hosseini, A modified Adomian decomposition method for singular initial value Emden-Fowler type equations. International Journal of Applied Mathematical Research, 5 (1) (2016), 69-72.

[18] A. Neamaty, B. Agheli, R. Darzi, Variational Iteration Method and He's Polynomials for Time-Fractional Partial Differential Equations, Progr. Fract. Differ. Appl., 1 (1) (2015), 47-55.

[19] M. Mirzazadeh, Z. Ayati, New homotopy perturbation method for system of Burgers equations, Alexandria Engineering Journal, 2016.

\section{RAHMAT DARZI}

Department of Mathematics

NEKA BRANCH

ISLAMIC AZAD University

NEKA

IRAN

E-mail address: r.darzi@iauneka.ac.ir

\section{Bahram Agheli}

Department of Mathematics

QAEMSHAHR BRANCH

ISLAMIC AzAD UNIVERSity

QAEMSHAHR

IRAN

E-mail address: b.agheli@qaemiau.ac.ir 